\title{
Fatores de risco para o desencadeamento do pé diabético*
}

\author{
Risk factors for developing diabetic foot
}

Factores de riesgo para el desencadenamiento del pie diabético

Julia Estela Willrich Boell ${ }^{1}$, Renata Mafra Ribeiro ${ }^{2}$, Denise Maria Guerreiro Vieira da Silva ${ }^{3}$

\footnotetext{
* Artigo originado do Trabalho de Conclusão de Curso Fatores de risco para o desencadeamento do pé diabético em uma comunidade de Florianópolis/SC apresentado ao curso de graduação em Enfermagem da Universidade Federal de Santa Catarina, em 2011.

${ }^{1}$ Educadora Física e Enfermeira, Mestre em Enfermagem. Florianópolis, SC, Brasil. E-mail: juliaestela_8@hotmail.com.

2 Enfermeira. Florianópolis, SC, Brasil. E-mail: renatamr2003@yahoo.com.br.

${ }^{3}$ Enfermeira, Doutora em Enfermagem. Professora do Departamento de Enfermagem do Centro de Ciências da Saúde da Universidade Federal de Santa Catarina. Bolsista produtividade do CNPq. Florianópolis, SC, Brasil. E-mail: denise querreiro@hotmail.com.
}

\section{RESUMO}

Objetivou-se identificar os fatores de risco para o desencadeamento do pé diabético. Estudo transversal, com amostra por conveniência, desenvolvido com 70 pessoas com diabetes melito (DM), cadastradas em três unidades básicas do município de Florianópolis/SC, no período de novembro de 2010 a maio de 2011. Foram coletados dados biométricos, das condições sociodemográficas, de saúde e doença e efetuada a avaliação dos pés. Os participantes tinham idade média de 66,17 anos e conviviam com a doença há menos de 10 anos (61,42\%). Como fatores de risco, identificaramse: idade avançada; tempo de diagnóstico do DM; baixa escolaridade; sobrepeso/obesidade; dieta inadequada; inatividade física; controle metabólico inadequado; falta de cuidados específicos com os pés; e hipertensão arterial. Averiguou-se que parte majoritária da população apresentou um ou mais fatores de risco que favorecem o aparecimento de complicações relativas aos pés.

Descritores: Diabetes Mellitus; Pé Diabético; Fatores de Risco; Atenção Primária à Saúde; Enfermagem.

\section{ABSTRACT}

The goal of the present study is to identify the risk factors for developing diabetic foot. A cross-sectional study, with a convenience sample, developed with 70 individuals with diabetes mellitus (DM), registered in three basic health units in the municipality of Florianópolis/SC, Brazil, in the period from November 2010 to May 2011. Biometric data was collected regarding their sociodemographic, health and illness conditions. An assessment of the feet was also carried out. The average participant age was 66.17 years and time with diagnosed disease was under ten years (61.42\%). The following risk factors were identified: advanced age; time of DM diagnosis; few years of schooling; overweight/obesity; inadequate diet; physical inactivity; inadequate metabolic control; lack of proper and specific foot care; and arterial hypertension. We conclude that the majority of the population presented one or more risk factors that favor the appearance of foot-related complications.

Descriptors: Diabetes Mellitus; Diabetic Foot; Risk Factors; Primary Health Care; Nursing.

\section{RESUMEN}

Se objetivó identificar los factores de riesgo para el desencadenamiento del pie diabético. Estudio transversal con muestra por conveniencia, desarrollado con 70 personas con diabetes mellitus (DM), registradas en tres unidades primarias del municipio de Florianópolis/SC, dese noviembre de 2010 a mayo de 2011. Fueron recolectados datos biométricos, de condiciones sociodemográficas, de salud y enfermedad y efectuada la evaluación de los pies. Los participantes tenían una media etaria de 66,17 años y convivían con la enfermedad desde menos que diez años (61,42\%). Como factores de riesgo, se identificaron: edad avanzada; tiempo de diagnóstico de la DM; baja escolarización; sobrepeso/obesidad; dieta inadecuada; inactividad física; control metabólico inadecuado; falta de cuidados específicos con los pies; e hipertensión arterial. Se averiguó que la parte mayoritaria de la población presentó uno o más factores de riesgo que favorecen el surgimiento de complicaciones relativas a los pies.

Descriptores: Diabetes Mellitus; Pie Diabético; Factores de Riesgo; Atención Primaria de Salud; Enfermería. 


\section{INTRODUÇÃo}

O Diabetes Mellitus (DM) é uma doença crônica não transmissível que provoca mudanças na vida das pessoas, necessita de cuidados específicos para o adequado manejo da doença, requer colaboração entre as pessoas com DM, familiares e profissionais de saúde ${ }^{(1)}$. Seu aparecimento pode estar relacionado a fatores hereditários ou ambientais. Demanda constante atenção à dieta, exercícios físicos, monitoramento da glicose e, em muitos casos, uso de medicação ${ }^{(2)}$. Em âmbito mundial o DM vem tomando grandes proporções e sua incidência cresce progressivamente, apresentando alta morbimortalidade. Atualmente existem 382 milhões de pessoas no mundo com DM, e estima-se um aumento de $55 \%$ de casos até o ano de 2035. O Brasil encontrase em quarto lugar no ranking dos 10 países com maior prevalência do diabetes, aproximadamente 12 milhões de brasileiros convivem com a doença ${ }^{(1)}$.

O diabetes é uma importante causa de óbito devido ao alto risco de desenvolvimento de complicações agudas e crônicas $^{(1)}$. É apontado como um dos principais responsáveis pelo desenvolvimento de insuficiência renal, retinopatia, doenças cardiovasculares, doença arterial coronariana e amputação de membros inferiores. As complicações macrovasculares, oriundas da doença de base, resultam em alterações nos vasos sanguíneos de médio e grosso calibre como a enfermidade aterosclerótica, com manifestações clínicas principalmente nas artérias coronárias, cerebrais e periféricas de extremidades inferiores ${ }^{(2)}$.

A neuropatia diabética (ND) é uma complicação que constitui importante problema de saúde pública, apresenta heterogeneidade de formas e manifestações clínicas. Engloba um conjunto de doenças que afetam diferentes partes do sistema nervoso, inclusive os nervos periféricos (sensório motores), autônomos e espinhais ${ }^{(3)}$. Sintomas como dormência, queimação, "pontadas" e "agulhadas", bem como a perda da sensibilidade térmica e dolorosa nos membros inferiores caracterizam o início da doença ${ }^{(2)}$.

Aliada à redução do fluxo sanguineo, a neuropatia nos pés, aumenta a chance do desenvolvimento de úlceras e eventual amputação de membros inferiores. Esses casos estão relacionados a pequenos traumas devido mais comumente ao uso de calçados inadequados, dermatoses e/ou manipulação incorreta dos pés e unhas ${ }^{(4)}$.

Pé diabético é o termo utilizado para denominar o aparecimento de pontos de perda da sensibilidade protetora plantar, podendo ou não evoluir com o aparecimento de úlceras como consequência da ND. As úlceras tendem a complicar com gangrena e infecção, devido à doença de base, a cicatrização dessas lesões pode apresentar comprometimento ${ }^{(5)}$.

Os principais fatores de risco apontados para o desencadeamento do pé diabético são: a neuropatia, a insuficiência vascular e a predisposição à infecção. Estudos apontam outros fatores que influenciam o desencadeamento do pé diabético, como: idade avançada, tipo e tempo de diagnóstico do DM, controle metabólico inadequado, tabagismo, alcoolismo, obesidade, hipertensão arterial e falta de bons hábitos higiênicos no cuidado com os pés ${ }^{(6-7)}$.

Além de a doença apresentar tais complicações que afetam a rotina da pessoa com DM e de quem a cerca, as complicações da doença também são onerosas para os sistemas de saúde em todo o mundo(1). Os profissionais assumem o compromisso de prestar assistência integral à população, identificando fatores de risco aos quais ela está exposta, intervindo de forma apropriada.

A avaliação dos pés das pessoas com DM de forma minuciosa e com frequência regular, pelos profissionais da saúde, ainda não parece ser uma realidade na atenção básica do nosso país. Neste sentido, desconhecemos a condição dos pés das pessoas com DM e quais os fatores de risco mais presentes em nosso contexto. Reconhecemos que o trabalho do enfermeiro é essencial, rastreando e monitorando os fatores de risco sugestivos desencadeadores do pé diabético ${ }^{(5)}$.

A relevância do tema escolhido e a escassez de estudos que abordam a temática na realidade brasileira justificam esse estudo, que tem por objetivo: identificar os fatores de risco para o desencadeamento do pé diabético.

\section{MÉTODO}

Estudo transversal, desenvolvido em três Unidades Básicas de Saúde (UBS) localizadas em uma comunidade da cidade de Florianópolis/SC-Brasil.

A população do estudo foi de 143 homens e mulheres com diagnóstico de DM tipo 1 ou 2, cadastradas no sistema de saúde das UBS, de acordo com informação fornecida ao iniciar a coleta de dados.

A amostra de conveniência foi constituída por 70 pessoas, com registro do diagnóstico de DM na UBS. Os critérios de inclusão dos sujeitos no estudo foram: ter diagnóstico de DM há mais de um ano e idade superior a 18 anos. Os critérios de exclusão adotados foram: não ter condições físicas ou emocionais de responder ao 
questionário; apresentar dificuldade de comunicação e/ou possuir déficit cognitivo, a partir de avaliação subjetiva das pesquisadoras.

Essas pessoas foram contatadas na própria UBS ao realizarem consulta de enfermagem ou médica, ou nos seus domicílios por indicação das enfermeiras e/ou agentes comunitários de saúde.

Foi aplicado um instrumento de coleta de dados reunindo: dados do prontuário coletados na UBS de referência (valores do último exame ou realizados até o ano de 2010 de: glicemia de jejum, hemoglobina glicada, colesterol - HDL e LDL - e triglicerídeos); dados sociodemográficos (idade, ocupação e escolaridade) dados sobre a condição saúde e doença (IMC, dieta, atividade física, consumo de cigarro, frequencia do consumo de bebida alcoólica, tempo do diagnóstico do DM, hipertensão arterial, pressão arterial, uso de hipoglicemiante oral, uso de insulina), além da avaliação dos pés.

Foram considerados como fatores de risco para o desenvolvimento do pé diabético no estudo: idade avançada; mais de 10 anos do diagnóstico de DM; baixa escolaridade; sobrepeso e obesidade; dieta inadequada; inatividade física; controle metabólico inadequado; hipertensão arterial; tabagismo; alcoolismo; e falta de cuidados com os pés, além do comprometimento da sensibilidade protetora plantar ${ }^{(5-7)}$.

A avaliação dos pés foi realizada seguindo os critérios determinados pelo Grupo Multiprofissional de Atendimento ao Diabético (GRUMAD) do Hospital Universitário - HU/UFSC ${ }^{(9)}$, incluindo: histórico em relação aos pés; inspeção dos pés e calçados; avaliação vascular do pé, dimensionada a partir do pulso pedioso e tibial posterior além do tempo de enchimento venoso e capilar; e avaliação neurológica, onde foram realizados os testes de sensibilidade tátil, térmica e dolorosa, usando algodão, cabo do diapasão e palito de madeira pontiagudo; percepção de vibração usando um diapasão de 128 hertz; força muscular tibial anterior e da panturrilha; reflexo aquileu, usando martelo neurológico e sensibilidade protetora plantar, através da sensação de pressão do monofilamento de $10 \mathrm{~g}$ aplicado à região plantar do pé num ângulo de 90 graus, através da técnica de resposta $\operatorname{sim} /$ não ao toque deste nas regiões: falanges distais do hálux e quinto pododáctilo e primeira e quinta cabeça dos metatarsos.

A coleta de dados ocorreu entre novembro de 2010 a maio de 2011, sendo agendada por contato diretos e/ou telefônico, realizada por duas pesquisadoras. As entrevistas tiveram duração média de 50 minutos.
Para uma maior confiabilidade, os dados foram duplamente digitados em planilha do Excel e realizada checagem automática de consistência e amplitude através do software EpiData 3.1. Os dados foram analisados utilizando estatística descritiva com uso da ferramenta computacional on-line Sestatnet ${ }^{\circledR 1}$.

A pesquisa obedeceu a Resolução no 196/96 e foi aprovada pelo Comitê de Ética em Pesquisa com Seres Humanos, processo no 959/10, e anuência da Prefeitura Municipal de Saúde de Florianópolis pela Comissão de Acompanhamento dos Projetos de Pesquisa em Saúde. Foi obtido o Termo de Consentimento Livre e Esclarecido e respeitados os princípios éticos de justiça, respeito à dignidade humana e beneficência; garantindo o sigilo e o anonimato; e respeitado o direito de desistir da participação do estudo a qualquer momento.

\section{RESULTADOS}

A idade média dos participantes do estudo foi de 66,17 anos (DP: 12,09), a maior frequência de escolaridade encontrada na população do estudo foi até a quarta série completa $(66,00 \%)$, e no que diz respeito ao perfil laboral dos participantes, a maioria referiu não possuir trabalho remunerado $(78,26 \%)$, podendo ser aposentado, pensionista ou não receber nada, sendo que apenas uma pessoa não respondeu a esta questão, conforme consta da Tabela 1.

No que diz respeito a frequência com que são realizados os cuidados não farmacológicos para um bom controle glicêmico e cuidados gerais com a saúde, 63 pessoas $(90,00 \%)$ relataram ter recebido orientação sobre a alimentação ideal, porém, grande parte delas não segue a dieta recomendada sempre, alegando seguir na maioria das vezes $(42,86 \%)$. Com relação a realização de atividades físicas regulares, $72,86 \%$ relatam que não costumam praticar (Tabela 2).

\footnotetext{
${ }^{1}$ SestatNet - Ensino-Aprendizagem de Estatística na Web. Disponivel em: http://www.sestatnet.ufsc.br/
} 
Tabela 1: Distribuição das variáveis sociodemográficas de pessoas com DM cadastrados na atenção básica de uma comunidade de Florianópolis, SC, Brasil, 2011.

\begin{tabular}{|c|c|c|}
\hline Variáveis & $\mathbf{n}$ & $\%$ \\
\hline \multicolumn{3}{|l|}{ Idade } \\
\hline $37-50$ & 5 & 7,14 \\
\hline $51-60$ & 20 & 28,57 \\
\hline $61-70$ & 15 & 21,42 \\
\hline $71-80$ & 24 & 34,28 \\
\hline Acima de 81 & 6 & 8,57 \\
\hline \multicolumn{3}{|l|}{ Ocupação } \\
\hline Não possui trabalho remunerado & 11 & 15,71 \\
\hline Possui trabalho remunerado & 16 & 22,86 \\
\hline Aposentado & 27 & 38,57 \\
\hline Pensionista & 15 & 21,43 \\
\hline Não respondeu a questão & 1 & 1,43 \\
\hline \multicolumn{3}{|l|}{ Escolaridade } \\
\hline Nunca estudou & 5 & 7,14 \\
\hline Nunca estudou, mas sabe ler e escrever & 1 & 1,43 \\
\hline Ensino fundamental & 51 & 72,85 \\
\hline Ensino médio & 5 & 7,14 \\
\hline Ensino superior & 7 & 10 \\
\hline Pós graduação & 1 & 1,43 \\
\hline
\end{tabular}

Tabela 2: Distribuição das variáveis conforme as condições de saúde e doença de pessoas com DM cadastrados na atenção básica de uma comunidade de Florianópolis, SC, Brasil, 2011.

\begin{tabular}{|c|c|c|c|c|}
\hline Variáveis & $\mathbf{n}$ & $\%$ & $\mathbf{X}$ & DP \\
\hline Índice de Massa Corpórea (IMC) & 70 & & 27.76 & 5.22 \\
\hline Baixo peso $(\leq 18,4)$ & 1 & 1,43 & & \\
\hline Normal $(18,5-24,9)$ & 22 & 31,42 & & \\
\hline Sobrepeso $(25-29,9)$ & 24 & 34,28 & & \\
\hline Obeso classe I $(30-34,9)$ & 17 & 24,28 & & \\
\hline Obeso classe II $(35-39,9)$ & 5 & 7,14 & & \\
\hline Obeso classe III $(\geq 40)$ & 1 & 1,43 & & \\
\hline \multicolumn{5}{|l|}{ Dieta } \\
\hline Sempre & 20 & 28,57 & & \\
\hline Na maioria das vezes & 30 & 42,86 & & \\
\hline Raramente & 9 & 12,86 & & \\
\hline Nunca & 8 & 11,43 & & \\
\hline Não recebeu orientação & 1 & 1,43 & & \\
\hline Não respondeu a questão & 2 & 2,86 & & \\
\hline \multicolumn{5}{|l|}{ Atividade física } \\
\hline Nunca & 51 & 72,86 & & \\
\hline 1 vez por semana & 2 & 2,86 & & \\
\hline 2 vezes por semana & 7 & 10 & & \\
\hline 3 vezes por semana ou mais & 10 & 14,29 & & \\
\hline \multicolumn{5}{|l|}{ Consumo de cigarro } \\
\hline Sim & 4 & 5,71 & & \\
\hline Não & 66 & 94,29 & & \\
\hline \multicolumn{5}{|l|}{ Frequência consumo de bebida alcoólica } \\
\hline Nunca & 60 & 85,71 & & \\
\hline Todos os dias & 3 & 4,29 & & \\
\hline Finais de semana & 4 & 5,71 & & \\
\hline Ocasionalmente & 3 & 4,29 & & \\
\hline
\end{tabular}

Outros hábitos importantes, referentes aos cuidados para evitar o desencadeamento de problemas de saúde consequentes da DM, são o uso demasiado de bebidas alcoólicas e fumo. Neste estudo, 94,29\% relataram não estar fumando no momento da entrevista, sendo que do total de entrevistados $21,43 \%$ já fumou. Referente ao consumo de álcool, $85,71 \%$ relataram não ingerir bebidas alcoólicas (Tabela 2 ).

$\mathrm{Na}$ Tabela 3 observa-se que a maioria dos entrevistados $(76,81 \%)$ convive com a doença há menos de 10 anos, porém a média de idade foi de 11,91 anos. A maioria $(64,14 \%)$ apresenta um bom controle glicêmico. Quanto à prevenção especificamente do pé diabético, podemos destacar a avaliação dos pés por profissional de saúde habilitado, onde apenas $35,81 \%$ dos entrevistados já havia realizado esta avaliação. Em relação à terapia farmacológica $82,85 \%$ faziam uso diário de hipoglicemiantes orais e $8,57 \%$ de insulina. 
Tabela 3: Distribuição dos dados relacionados ao DM de pessoas cadastradas na atenção básica de uma comunidade de Florianópolis, SC, Brasil, 2011.

\begin{tabular}{|c|c|c|c|c|}
\hline Variáveis & $\mathbf{n}$ & $\%$ & $\mathbf{X}$ & DP \\
\hline Tempo de DM & & & 11,91 & 9,54 \\
\hline Entre 1 e 10 anos & 43 & 61,43 & & \\
\hline Entre 11 e 20 anos & 19 & 27,14 & & \\
\hline Entre 21 e 30 anos & 3 & 4,28 & & \\
\hline Entre 31 e 40 anos & 4 & 5,71 & & \\
\hline Não respondeu a questão & 1 & 1,43 & & \\
\hline \multicolumn{5}{|l|}{ Glicemia de jejum* } \\
\hline Abaixo de $90 \mathrm{mg} / \mathrm{dl}$ & 2 & 3,77 & & \\
\hline Entre 91 e 130 mg/dl & 32 & 60,37 & & \\
\hline Entre 131 e 230 mg/dl & 14 & 26,41 & & \\
\hline Entre 231 e 331 mg/dl & 5 & 9,43 & & \\
\hline \multicolumn{5}{|l|}{ Hemoglobina Glicada } \\
\hline Entre 5,9 e 7,0 & 11 & 36,66 & & \\
\hline Entre 7,1 e 9,0 & 12 & 40 & & \\
\hline Entre 9,1 e 11,0 & 3 & 10 & & \\
\hline Entre 11,1 e 13,5 & 4 & 13,33 & & \\
\hline \multicolumn{5}{|l|}{ Hipertensão Arterial } \\
\hline Sim & 42 & 60 & & \\
\hline Não & 28 & 40 & & \\
\hline Pressão Arterial Sistólica & & & 122 & 17,32 \\
\hline Entre 90 e 110 & 27 & 38.57 & & \\
\hline Entre 120 e 140 & 35 & 50 & & \\
\hline Entre 150 e 170 & 8 & 11.42 & & \\
\hline Pressão Arterial Diastólica & & & 76,57 & 10,19 \\
\hline Entre 60 e 80 & 59 & 84.44 & & \\
\hline Entre 90 e 100 & 11 & 15.71 & & \\
\hline \multicolumn{5}{|l|}{ Hipoglicemiante oral } \\
\hline Sim & 58 & 82.85 & & \\
\hline Não & 12 & 17.14 & & \\
\hline \multicolumn{5}{|l|}{ Insulina } \\
\hline Sim & 6 & 8.57 & & \\
\hline Não & 64 & 91.42 & & \\
\hline \multicolumn{5}{|l|}{ Avaliação dos pés por profissional da saúde } \\
\hline Sim & 25 & 35.71 & & \\
\hline Não & 45 & 64.29 & & \\
\hline \multicolumn{5}{|l|}{ Formato do corte das unhas } \\
\hline Reta & 22 & 31.42 & & \\
\hline Arredondada & 42 & 60 & & \\
\hline Não respondeu a pergunta & 6 & 8.57 & & \\
\hline \multicolumn{5}{|l|}{ Úlcera prévia } \\
\hline Nunca & 60 & 85.71 & & \\
\hline $1 \mathrm{vez}$ & 7 & 10 & & \\
\hline 2 vezes & 2 & 2.85 & & \\
\hline 3 vezes & 1 & 1.43 & & \\
\hline
\end{tabular}

Mais da metade dos entrevistados $(60,00 \%)$ referiu ter hipertensão arterial quando questionado se possui outra doença além da DM. Foi ainda verificada a pressão arterial no momento da entrevista, e como pode ser observado na Tabela 3, a média da Pressão Arterial Sistólica (PAS) ficou em 122 mmHg e da Pressão Arterial Diastólica (PAD) em $76.57 \mathrm{mmHg}$, sendo assim, apesar de grande parte das pessoas possuir hipertensão, estas apresentavam um bom controle da mesma.

Referente ao controle glicêmico dos participantes foram utilizados apenas os dados registrados nos prontuários a partir de janeiro de 2010. Foram obtidos dados de 38 pessoas, uma vez que 17 pessoas não apresentaram registro de quaisquer exames em seu prontuário e o demais participantes, 15 pessoas, apresentaram dados de registros muito anteriores (entre 2002 e 2009), sendo desconsiderados nesse estudo.

$\mathrm{Na}$ avaliação neurológica dos pés, observou-se que $80,00 \%$ das pessoas possuíam a sensibilidade protetora plantar preservada, e 17,14\% apresentaram alterações na sensibilidade, aliada a presença de alteração nos pés, tais como: calos, deformidades e formação de ulceração por estresse repetitivo. Um participante tinha amputação do hálux direito. A Tabela 4 apresenta a classificação de risco para pé diabético dos participantes. 
Tabela 4: Classificação de risco para pé diabético de pessoas com DM cadastrados na atenção básica de uma comunidade de Florianópolis, SC, Brasil, 2011.

\begin{tabular}{|c|c|c|c|}
\hline Variáveis & Risco & $\mathbf{n}$ & $\%$ \\
\hline \multicolumn{4}{|l|}{ Risco geral } \\
\hline Neuropatia ausente & 0 & 56 & 80 \\
\hline Neuropatia presente & 1 & 12 & 17,14 \\
\hline Neuropatia presente, sinais de doença vascular periférica e/ou deformidade dos pés & 2 & 1 & 1,43 \\
\hline Amputação/úlcera prévia & 3 & 1 & 1,43 \\
\hline
\end{tabular}

Constatou-se que $20 \%$ dos indivíduos apresentaram algum grau de neuropatia, dentre o restante da população, foram identificados um ou mais fatores de risco.

\section{DISCUSSÃO}

As características sociodemográficas da população do estudo são semelhantes ao encontrado em outros estudos $^{(10-11)}$, especialmente em relação à idade, escolaridade e atividade laboral, uma vez que são pessoas com mais de 60 anos, com a média de 11 anos de doença, além de apresentarem um baixo nível de escolaridade.

A baixa escolaridade vem sendo apontada como fator de risco importante em alguns estudos ${ }^{(5,12)}$, os quais indicam que o baixo grau de instrução é um forte aliado ao desenvolvimento de complicações, por influenciar a capacidade dos indivíduos de assimilarem os conhecimentos sobre a doença e a importância dada ao controle do DM, incluindo aí os cuidados preventivos às complicações.

Estudos relacionam o tempo transcorrido desde o início do DM com a ocorrência do pé diabético, revelando em torno de 10 anos de convivência com a doença para o desencadeamento do mesmo ${ }^{(5-7)}$. Desta forma, como a maioria da população deste estudo encontra-se entre um e 10 anos desde o diagnóstico, observamos a presença de tal fator como relevante para o desencadeamento da neuropatia periférica, apesar de termos identificado que $80 \%$ dos participantes não apresentavam alteração nos pés.

Além dos fatores de risco que não são modificáveis, como a idade e o tempo transcorrido, há outros que estão relacionados a hábitos de vida que poderiam ser modificados. No entanto, como vimos no presente estudo, as pessoas têm dificuldade de seguir o tratamento e manter um controle glicêmico adequado como mostram os resultados da hemoglobina glicada. Os resultados da glicemia em jejum foram melhores e isto pode estar relacionado ao fator cultural, no qual as pessoas tendem a ser mais cuidadosas com a dieta dois ou três dias antes da coleta do referido exame, obtendo um resultado que nem sempre expressa sua real condição, como é possível através da hemoglobina glicada, o que é condizente com outros estudos ${ }^{(6,13)}$.

Ainda com relação ao controle glicêmico, é recomendado executar o teste da hemoglobina glicada trimestralmente para os indivíduos que não mantêm controle adequado, ao passo que para aqueles que têm adesão ao tratamento e apresentam estabilidade, recomenda-se a execução do referido exame no mínimo duas vezes ao $a n o^{(2)}$. Confrontando a situação encontrada, na qual a partir da leitura dos prontuários da população em questão, não observamos esta regularidade, evidenciada pela desatualização desses exames.

Mesmo com as pessoas referindo que realizam a dieta na maioria das vezes, esta situação nem sempre expressa a realidade, como mostra estudo que refere a aderência ao tratamento, nos quais há também uma baixa adesão ao tratamento não medicamentoso(13-14). Outro aspecto que nos possibilita este questionamento é que grande parte das pessoas do grupo estudado estavam com peso acima do indicado. Este índice foi semelhante a um estudo encontrado, no qual a média de IMC também encontrava-se com valores acima do parâmetro recomendado ${ }^{(14)}$.

Não é comum encontrar autores que relacionem especificamente a prevenção do desenvolvimento do pé diabético com a aderência aos cuidados não farmacológicos, mas é consenso que estes cuidados favorecem a manutenção de peso evitando assim, a obesidade, auxiliando no controle glicêmico e indiretamente também na prevenção das diversas comorbidades causadas pelo $\mathrm{DM}^{(14)}$. É destacado em outros estudos que grande parte dos sujeitos tinham sobrepeso ou obesidade, corroborando com os resultados deste estudo e apontando como um importante fator de $\operatorname{risco}^{(6,9)}$.

No que se refere ao uso de bebidas alcoólicas e ao fumo, houve número reduzido que referiram tais hábitos incorporados, fato que difere de diversos estudos onde o hábito de consumir bebidas alcoólicas e tabaco encontrava-se presente ${ }^{(6,15)}$. É relevante salientar a cessação de tais hábitos como medida de prevenção para complicações e busca de hábitos saudáveis de vida. 
Neste estudo, a grande maioria não fazia nenhum exercício físico, sendo este valor superior ao encontrado em outro estudo(15), o qual $62,1 \%$ dos participantes com DM eram sedentários. Destacamos que a associação entre a realização de exercício físico e dieta são elementos essenciais para o bom controle glicêmico e, deste modo, para a prevenção do pé diabético.

Fazer uso da medicação hipoglicemiante é outro elemento importante do tratamento do diabetes, que contribui para o melhor controle glicêmico, favorecendo a prevenção do pé diabético. Os achados do presente estudo mostraram um número expressivo de pessoas em uso de hipoglicemiante oral, diferentemente dos achados de outra pesquisa ${ }^{(5)}$, que encontrou um percentual inferior $(53,5 \%)$. Por outro lado, o uso de insulina entre os participantes deste estudo foi bastante inferior ao daquele.

A hipertensão arterial, quando associada a outros fatores como obesidade, dislipidemia e doença arterial coronariana, sem o adequado tratamento, pode levar ao aparecimento e progressão das complicações crônicas do DM, como o desenvolvimento de úlceras neuroisquêmicas ${ }^{(1-2)}$. Entretanto, a hipertensão isolada não pode ser considerada fator de risco relevante para o desenvolvimento do pé diabético.

Considerando os parâmetros atuais de medida de pressão arterial(16), a média da PA tanto sistólica quanto diastólica está dentro dos parâmetros, uma vez que é considerada como hipertensão a pressão acima de $140 / 90 \mathrm{~mm} / \mathrm{Hg}$. No entanto, $60 \%$ dos entrevistados referiu ter hipertensão arterial sistêmica (HAS) quando questionado a respeito das doenças concomitantes ao DM, logo, encontramos valores dentro dos parâmetros recomendados.

Com relação à classificação de risco para o pé diabético, os achados deste estudo, corroboram com outro estudo(17), no qual $74 \%$ da população avaliada através do monofilamento $10 \mathrm{~g}$ mantiveram a sensibilidade protetora plantar preservada. Já no estudo de Ochoa-Vigo et $\mathrm{al}^{(5)}$, 7,9\% dos avaliados apresentaram perda da sensibilidade protetora plantar, e também não foi encontrada alteração da sensibilidade aliada a doença vascular ou deformidade e tampouco amputação, diferindo dos dados encontrados neste estudo. A questão da ocorrência de úlcera prévia conflui com outro estudo(18), atentando-se para o fato que a ulceração anterior está relacionada ao aumento do risco de amputação(10). $^{(10}$.

No que se refere ao corte de unhas, esse nem sempre era feito de maneira adequada, como também foi apontado em outro estudo(11), em que $65 \%$ das pessoas realizava-o de maneira não recomendada. Apesar de termos encontrado uma situação mais favorável, ainda assim, é surpreendente como um cuidado tão básico e conhecido entre os profissionais não é adotado por um número expressivo de pessoas, uma vez que esta situação pode favorecer lesões nas laterais das unhas contribuindo para a ocorrência de unha encravada.

Menos de $40 \%$ da amostra já teve seus pés avaliados por profissional da saúde habilitado ao menos uma vez após diagnóstico de DM. Avaliamos que este é um percentual muito baixo, já que de acordo com a $\mathrm{ADA}^{(2)}$ todas as pessoas diagnosticadas com DM tipo 2 devem ser avaliadas quanto a ocorrência de neuropatia diabética no momento do diagnóstico e após, anualmente.

A partir dessa situação torna-se evidente que, parte da população desse estudo desconhecia os cuidados básicos de higiene e cuidado com os pés, essenciais para a prevenção de alterações que podem vir a desencadear ulcerações e/ou feridas.

\section{CONCLUSÕES}

Com base nos resultados obtidos neste estudo, concluímos que a população estudada apresentou como fatores de risco para o desencadeamento do pé diabético: idade avançada; tempo de diagnóstico do DM; baixa escolaridade; sobrepeso e obesidade; dieta inadequada; diagnóstico de hipertensão arterial; inatividade física; controle metabólico inadequado; e falta de cuidados específicos com os pés.

Dessa forma, averiguou-se que parte majoritária da população deste estudo apresentou um ou mais fatores de risco que favorecem o aparecimento de complicações relativas ao pé. Acredita-se que tal situação possa ser contida e/ou prevenida na atenção básica de saúde, mediante atuação da enfermagem junto à equipe multidisciplinar, proporcionando às pessoas com diabetes um cuidado especializado, de forma a promover o autocuidado e contribuir para melhora da qualidade de vida das pessoas com DM e promoção da saúde.

Neste sentido, evidencia-se, a necessidade de uma avaliação dos pés de pessoas com DM periodicamente como parte da consulta de enfermagem, e pelos demais profissionais de saúde. Consideramos uma medida fundamental na identificação dos fatores de risco que podem ser modificados, o que, consequentemente, reduzirá o risco de ulceração e amputação de membros inferiores nas pessoas com diabetes. 
Identificamos como fragilidade do estudo o fato de que muitos exames laboratoriais não estavam registrados nos prontuários, o que demonstra também uma fragilidade no sistema de saúde que pode ser avaliado em dois aspectos: déficit de assistência de

\section{REFERÊNCIAS}

1. International Diabetes Federation. IDF Diabetes Atlas: sixth edition [Internet]. 2013 [acesso em: 30 jun 2014]. Disponível em:

http://www.idf.org/sites/default/files/EN_6E_Atlas_Full_0.pdf. 2. American Diabetes Association. Standards of Medical Care in Diabetes-2014. Diabetes Care [Internet]. 2014 [acesso em: 30 jun 2014];37 Suppl 1:S14-80. Disponível em:

http://dx.doi.org/10.2337/dc14-S014.

3. Lucas LPP, Barichello E, Zuffi FB, Barbosa MH. A percepção dos portadores de Diabetes Mellitus tipo 2 em relação à amputação. Rev. Eletr. Enf. [Internet]. 2010 [acesso em: 30 jun 2014];12(3):535-8. Disponível em: http://dx.doi.org/10.5216/ree.v12i3.6005.

4. Amaral AS, Tavares DMS. Cuidados com os pés: conhecimento entre pessoas com diabetes mellitus. Rev. Eletr. Enf. [internet]. 2009 [acesso em: 30 jun 2014];11(4):801-10. Disponível em: http://www.fen.ufg.br/fen_revista/v11/n4/pdf/v11n4a05.pdf. 5. Ochoa-Vigo K, Torquato MTCG, Silvério IAS, Queiroz FA; DeLa-Torre-Ugarte-Guanilo MC, Pace AE. Caracterização de pessoas com diabetes em unidades de atenção primária e secundária em relação a fatores desencadeantes do pé diabético. Acta paul. enferm. [Internet]. 2006 [acesso em: 30 jun 2014];19(3):296303. Disponível em: http://dx.doi.org/10.1590/S010321002006000300007.

6. Vieira-Santos ICR, Souza WV, Carvalho EF, Medeiros MCWC, Nóbrega MGL, Lima PMS. Prevalência de pé diabético e fatores associados nas unidades de saúde da família da cidade do Recife, Pernambuco, Brasil, em 2005. Cad Saude Publica [Internet]. 2008 [acesso em: 30 jun 2014];24(12):2861-70. Disponível em: http://dx.doi.org/10.1590/S0102-311X2008001200015.

7. Santos VP, Silveira DR, Caffaro RA. Risk factors for primary major amputation in diabetic patients. Sao Paulo Med J [Internet]. 2006 [acesso em: 30 jun 2014]; 124(2):66-70. Disponível em: http://dx.doi.org/10.1590/S151631802006000200004.

8. Tavares DMS, Dias FA, Araújo LR, Pereira GA. Perfil de clientes submetidos a amputações relacionadas ao diabetes mellitus. Rev Bras Enferm [Internet]. 2009 [acesso em: 30 jun 2014];62(6):825-30. Disponível em: http://dx.doi.org/10.1590/S0034-71672009000600004. 9. Sandoval RCB. Grupo multiprofissional de atendimento ao diabético/UFSC. Avaliação dos pés de pessoas com diabetes mellitus. Florianópolis: UFSC; 2004.

10. Assumpção EC, Pitta GB, Macedo ACL, Mendonça GB Albuquerque LCA, Lyra LCB et al. Comparação dos fatores de risco para amputações maiores e menores em pacientes diabéticos de um Programa de Saúde da Família. J. vasc. bras. [Internet]. 2009 [acesso em: 30 jun 2014];8(2):133-8. Disponível em: http://dx.doi.org/10.1590/S167754492009000200006

11. Cosson ICO, Ney-Oliveira F, Adan LF. Avaliação do conhecimento de medidas preventivas do pé diabético em pacientes de Rio Branco, Acre. Arq Bras Endocrinol Metabol [Internet]. 2005 [acesso em: 30 jun 2014];49(4):548-56. Disponível em: http://dx.doi.org/10.1590/S000427302005000400013.

12. Rocha RM, Zanetti ML, Santos MA. Comportamento e conhecimento: fundamentos para prevenção do pé diabético. Acta paul. enferm. [Internet]. 2009 [acesso em: 30 jun 2014];22(1):17-23. Disponível em: http://dx.doi.org/10.1590/S0103-21002009000100003. 13. Compeán Ortiz LG, Gallegos Cabriales EC, González González JG, Gómez Meza MV. Self-Care Behaviors and Health saúde especializada e exames para controle metabólico, ou simples falta de registro daqueles pacientes que fazem parte do tratamento e realizam os exames por meio de convênios de saúde ou particular.

Indicators in Adults with Type 2 Diabetes. Rev Lat Am Enfermagem [Internet]. 2010 [acesso em: 30 jun 2014];18(4):675-80. Disponível em: http://dx.doi.org/10.1590/S0104-11692010000400003. 14. Boas LCGV, Foss MC, Foss-Freitas MC, Torres HC, Monteiro LZ, Pace AE. Adesão à dieta e ao exercício físico das pessoas com diabetes mellitus. Texto Contexto Enferm [Internet]. 2011 [acesso em: 30 jun 2014];20(2):272-9. Disponível em: http://dx.doi.org/10.1590/S0104-07072011000200008. 15. Lima LM, Schwartz E, Muniz RM, Zillmer JGV, Ludtke I. Perfil dos usuários do Hiperdia de três unidades básicas de saúde do sul do Brasil. Rev Gaucha Enferm [Internet]. 2011 [acesso em: 30 jun 2014];32(2):323-9. Disponível em: http://dx.doi.org/10.1590/S1983-14472011000200016. 16. Sociedade Brasileira de Cardiologia; Sociedade Brasileira de Hipertensão; Sociedade Brasileira de Nefrologia. VI Diretrizes Brasileiras de Hipertensão. Arq Bras Cardiol [Internet]. 2010 [acesso em: 30 jun 2014];95(1 Supl 1):I-III. Disponível em: http://dx.doi.org/10.1590/S0066-782X2010001700001. 17. Araujo MM, Alencar AMPG. Pé de risco para o desenvolvimento de ulcerações e amputações em diabéticos. Rev. RENE. [Internet]. 2009 [acesso em: 30 jun 2014];10(2):19-28. Disponível em: http://www.revistarene.ufc.br/revista/index.php/revista/article/vi ew/470.

18. Cisneros LL, Gonçalves LAO. Educação terapêutica para diabéticos: os cuidados com os pés na realidade de pacientes e familiares. Cien Saude Colet [Internet]. 2011 [acesso em: 30 jun 2014];16 Supl 1:1505-14. Disponível em: http://dx.doi.org/10.1590/S1413-81232011000700086.

Artigo recebido em 26/09/2012. Aprovado para publicação em 28/01/2014. Artigo publicado em 30/06/2014. 